\title{
On the box-counting dimension of potential singular set for suitable weak solutions to the 3D Navier-Stokes equations
}

\author{
Yanqing Wang* and Gang $\mathrm{Wu}^{\dagger}$
}

\begin{abstract}
In this paper, we are concerned with the upper box-counting dimension of the set of possible singular points in space-time of suitable weak solutions to the 3D Navier-Stokes equations. By taking full advantage of the pressure $\Pi$ in terms of $\nabla \Pi$ in equations, we show that this upper box dimension is at most $135 / 104(\approx 1.30)$, which improves the known upper box-counting dimension $95 / 63(\approx 1.51)$ in Koh et al. 9, J. Differential Equations, 261: 3137-3148, 2016], 45/29( $\approx 1.55)$ in Kukavica et al. 11, Nonlinearity 25: $2775-2783,2012$ ] and $135 / 82(\approx 1.65)$ in Kukavica [10, Nonlinearity 22: 2889-2900, 2009].
\end{abstract}

MSC(2000): 35B65, 35D30, 76D05

Keywords: Navier-Stokes equations; suitable weak solutions; box-counting dimension

\section{Introduction}

We consider the following incompressible Navier-Stokes system in dimension 3

$$
\left\{\begin{array}{l}
u_{t}-\Delta u+u \cdot \nabla u+\nabla \Pi=0 \\
\operatorname{div} u=0 \\
\left.u\right|_{t=0}=u_{0}
\end{array}\right.
$$

where $u$ stands for the flow velocity field, the scalar function $\Pi=-\Delta^{-1} \partial_{i} \partial_{j}\left(u_{i} u_{j}\right)$ represents the pressure. The initial velocity $u_{0}$ satisfies $\operatorname{div} u_{0}=0$.

In his seminal work [13], Leray constructed the global weak solutions to the tridimensional Navier-Stokes equations, namely, for a divergence-free vector field $u_{0} \in L^{2}\left(\mathbb{R}^{3}\right)$, there exists a weak solution $(u, \Pi)$ such that $u \in L^{\infty}\left(0, T ; L^{2}\left(\mathbb{R}^{3}\right)\right) \cap L^{2}\left(0, T ; H^{1}\left(\mathbb{R}^{3}\right)\right)$ to the system (1.1). It follows from the interpolation inequality that $u \in L^{p}\left(0, T ; L^{q}\left(\mathbb{R}^{3}\right)\right)$ with $\frac{2}{p}+$ $\frac{3}{q}=\frac{3}{2}, \quad 2 \leq p \leq \infty$. Solonnikov [25] and Giga and Sohr [8] proved the following regularity

${ }^{*}$ Department of Mathematics and Information Science, Zhengzhou University of Light Industry, Zhengzhou, Henan 450002, P. R. China Email: wangyanqing20056@gmail.com

${ }^{\dagger}$ School of Mathematical Sciences, University of Chinese Academy of Sciences, Beijing 100049, P. R. China Email: wugangmaths@gmail.com 
of the finite energy weak solutions

$$
\begin{aligned}
& \nabla \Pi \in L_{t}^{s} L_{x}^{q} \quad \text { with } \quad \frac{2}{s}+\frac{3}{q}=4, \quad \Pi \in L_{t}^{s} L_{x}^{j} \quad \text { with } \quad \frac{2}{s}+\frac{3}{j}=3, \\
& u \in L_{t}^{m} L_{x}^{n} \quad \text { with } \quad \frac{2}{m}+\frac{3}{n}=2, \quad m \geq s, \quad n>q, 1<s, q<\infty .
\end{aligned}
$$

A priori estimates of solutions to the Navier-Stokes system in $\mathbb{R}^{3}$ such as $u \in L_{t}^{1} L_{x}^{\infty}$, $(-\Delta)^{\frac{\alpha}{2}} \nabla^{d} u \in L_{t}^{4 /(d+\alpha+1), \infty} L_{x}^{4 /(d+\alpha+1), \infty}(\alpha \in[0,2), d \geq 1), \nabla^{s} u \in L_{t}^{\frac{2}{2 s-1}} L_{x}^{2}$ can be found in [4, 5, 7, 29] and references therein.

However, the regularity of weak solutions mentioned above can not lead to the full regularity of weak solutions. The partial regularity result of weak solutions is also originated from Leray in [13], where it was shown that one dimension Lebesgue measure of the set of the potential time singular points for the weak solutions to 3D Navier-Stokes equations is zero. This result was improved by Scheffer in [20], where he showed that the set of possible time singular points has 1/2-dimensional measure zero. In 1970s, Scheffer [21 23] also considered the potential space-time singular points set of solutions to the Navier-Stokes equations via introducing the suitable weak solutions and proved that the Hausdorff dimension of the space-times singular points set of suitable weak solutions of the 3D Navier-Stokes equations is at most $5 / 3$. The so-called suitable weak solutions is a kind of weak solutions meeting the local energy inequality. A point is said to be a regular point to the Navier-Stokes equations (1.1) if one has the $L^{\infty}$ bound of $u$ in some neighborhood of this point. The remaining points are called singular point and denoted by $\mathcal{S}$. In this direction, the celebrated work is that one dimensional Hausdorff measure of the possible space-time singular points set of suitable weak solutions to the 3D Navier-Stokes equations is zero, which was proved by Caffarelli, Kohn, Nirenberg in [1]. The Caffarelli-Kohn-Nirenberg theorem completely rests on the following $\varepsilon$-regularity criterion: there is an absolute constant $\varepsilon$ such that, if

$$
\limsup _{r \rightarrow 0} \frac{1}{r} \iint_{Q(r)}|\nabla u|^{2} d x d t \leq \varepsilon
$$

then $(0,0)$ is a regular point, where $Q(r):=B(r) \times\left(-r^{2}, 0\right)$ and $B(r)$ denotes the ball of center 0 and radius $r$. Alternative approaches to Caffarelli-Kohn-Nirenberg theorem and its generalization were presented in several works (see, e.g., [2, 3, 10 12, 14 16, 19, 26 28, 30, 31]).

Notice that Hausdorff dimension is a kind of fractal dimension. Another widely used fractal dimension is box-counting or box dimension (Minkowski dimension). As pointed out by Falconer in [6], box dimension's popularity is largely due to its relative ease of mathematical calculation and empirical estimation, the definition goes back at least to the 1930s and it has been variously termed Kolmogorov entropy, entropy dimension, capacity dimension, metric dimension, logarithmic density and information dimension. Extensive study on the box dimension can be found in [6]. The definition of box dimension is via lower box dimension and upper box dimension. It is valid that Hausdorff dimension is less than upper box dimension (see e.g. [6]). For convenience, in what follows, box dimension means the upper box dimension. The fractal upper box dimension of potential time singular points set of finite energy weak solutions to the 3D Navier-Stokes system is at most $1 / 2$, which was proved by Robinson and Sadowski in [17]. By means of $\varepsilon$-regularity criterion proved in [12, 14], Robinson and Sadowski [18] obtained that the fractal upper box dimension of 
possible space-time singular points set of suitable weak solutions to the three-dimensional Navier-Stokes equations is at most $5 / 3$. It was shown that the parabolic fractal dimension of the singular points set is less than or equal to $135 / 82(\approx 1.65)$ by Kukavica in [10]. Later, Kukavica and Pei [11] improved this upper box dimension to $45 / 29(\approx 1.55)$. Very recently, Koh and Yang [9] proved that the fractal upper box dimension of the set of potential singular points of suitable weak solutions in the Navier-Stokes equations is bounded by $95 / 63(\approx 1.51)$. The purpose of this paper is to refine the fractal upper box dimension of potential singular points set to system (1.1). Before we state the main theorems of this paper, we present the definition of upper box-counting dimension.

Definition 1.1. The (upper) box-counting dimension of a set $X$ is usually defined as

$$
d_{b o x}(X)=\limsup _{\epsilon \rightarrow 0} \frac{\log N(X, \epsilon)}{-\log \epsilon},
$$

where $N(X, \epsilon)$ is the minimum number of balls of radius $\epsilon$ required to cover $X$.

Theorem 1.1. The (upper) box dimension of the set of possible singular points in spacetime of suitable weak solutions to the 3D Navier-Stokes equations (1.1) is at most 135/104( $\approx$ $1.30)$.

Remark 1.1. This improves the previous box dimension of potential singular set of suitable weak solutions to the tri-dimensional Navier-Stokes equations obtained in [9 11, 18].

As calculating the Hausdorff dimension of singular points set of suitable weak solutions to the Navier-Stokes equations, the size of potential singular points set in terms of boxcounting measure strongly depends on the $\varepsilon$-regularity criterion. To show Theorem 1.1, we prove the following result.

Theorem 1.2. Suppose that the pair $(u, \Pi)$ is a suitable weak solution to (1.1). Then $|u|$ can be bounded by 1 in some neighborhood of $\left(x_{0}, t_{0}\right)$ provided the following condition holds,

$$
\iint_{\tilde{Q}(r)}|\nabla u|^{2}+|u|^{10 / 3}+\left|\Pi-\bar{\Pi}_{\tilde{B}(r)}\right|^{5 / 3}+|\nabla \Pi|^{5 / 4} d x d t \leq r^{135 / 104} \varepsilon_{1},
$$

for an absolute constant $\varepsilon_{1}>0$.

The notations used here can be found at the end of this section.

Next, we give several remarks about this theorem.

Remark 1.2. Theorem 1.2 is an improvement of corresponding results proved by Kukavica and Pei in [11].

Remark 1.3. The proof of Theorem 1.2 follows an approach utilized in [9]. Compared with the proof of Koh and Yang [9], first, notice that the appearance of the pressure in equations is in terms of $\nabla \Pi$ rather than $\Pi$, thence, we can always replace $\Pi$ by $\Pi-\bar{\Pi}_{\Omega}$. Based on this, we could make full use of better decay estimate of pressure $\Pi-\bar{\Pi}_{\Omega}$ than that of [9] (see Lemma 2.2 in Section 22). This is partially motivated by the recent work in [15], where the authors used this trick to study the partial regularity of suitable weak solutions to the Navier-Stokes equations with fractional dissipation $(-\Delta)^{\alpha}$ in the case $\alpha=3 / 4$. Second, in the spirit of [11], we will utilize the quantity $\|\nabla \Pi\|_{L_{t, x}^{5 / 4}}^{5 / 4}$ bounded by the initial energy as widely as possible since the scaling of $\|\nabla \Pi\|_{L_{t, x}^{5 / 4}}^{5 / 4}$ is better than that of $\|\Pi\|_{L_{t, x}^{5 / 3}}^{5 / 3}$. This enables us to obtain that box dimension is at most $180 / 131(\approx 1.37)$. 
Remark 1.4. Based on referees' crucial comments involving inequality (3.6) and a very recent literature [16], we realized that there exists an appropriate interpolation inequality (2.3) for estimating the box dimension. In comparison with inequality (2.2) of Koh and Yang in [9], the inequality (2.3) allows us to take full advantage of quantity $\|\nabla u\|_{L^{2}(Q(r))}^{2}$ in (1.4). Though both $\|u\|_{L^{\infty, 2}(Q(r))}^{2}$ and $\|\nabla u\|_{L^{2}(Q(r))}^{2}$ have the same scaling, $\|u\|_{L^{\infty, 2}(Q(r))}^{2}$ appearing in (1.4) seems to be inappropriate in discussion of estimating the singular points set, that is, $\|\nabla u\|_{L^{2}(Q(r))}^{2}$ is more useful than $\|u\|_{L^{\infty, 2}(Q(r))}^{2}$ in our arguments. This further helps us to improve the box dimension from 180/131 to 135/104.

Remark 1.5. To the knowledge of the authors, the strategy that one applies the interior estimate of harmonic function to the pressure $\Pi-\bar{\Pi}_{\Omega}$ is due to [24, Lemma 2.1, p.222]. Lemma 2.2 proved in [24] combined with the proof of [9] yields that the upper box dimension is bounded by $635 / 441(\approx 1.44)$.

Remark 1.6. A combination of the idea of [9] and the quantity $\|\nabla \Pi\|_{L_{t, x}^{5 / 4}}^{5 / 4}$ as [11] implies that, the upper box dimension of singular points set is at most $75 / 51(\approx 1.47)$.

Remark 1.7. The upper bound on the Hausdorff dimension of the potential space-time singular points of suitable weak solutions to the generalized Navier-Stokes equations with $(-\Delta)^{\alpha}$ in the case $3 / 4 \leq \alpha<1$ is obtained in [15, 26]. An interesting issue is to estimate the box-dimension of singular points set of suitable weak solutions of the fractional NavierStokes equations in the same case.

With Theorem 1.2 in hand, we can present the proof of Theorem 1.1 .

Proof of Theorem 1.1. It follows from the definition of box-counting dimension that if $\delta<$ $d_{\text {box }}(\mathcal{S})$, there exists a sequence $\epsilon_{j} \rightarrow 0$ such that

$$
N\left(\mathcal{S}, \epsilon_{j}\right)>\epsilon_{j}^{-\delta}
$$

We proceed by contradiction below. We assume that $d_{\text {box }}(\mathcal{S})>135 / 104$, then we can choose a constant $d$ such that $180 / 131<d<d_{\text {box }}(\mathcal{S})$. Thus, there exists a decreasing sequence $\epsilon_{j} \rightarrow 0$ such that

$$
N\left(\mathcal{S}, \epsilon_{j}\right)>\epsilon_{j}^{-d}
$$

Let $\left(x_{i}, t_{i}\right)_{i=1}^{N\left(\mathcal{S}, \epsilon_{j}\right)}$ be a collection of $\epsilon_{j}$-separated points in $\mathcal{S}$. According to Theorem 1.2, for any $\left(x_{i}, t_{i}\right) \in \mathcal{S}$, we get

$$
\int_{t_{i}-\epsilon_{j}^{2}}^{t_{i}} \int_{\tilde{B}_{i}\left(\epsilon_{j}\right)}|\nabla u|^{2}+|u|^{10 / 3}+\left|\Pi-\bar{\Pi}_{\tilde{B}_{i}\left(\epsilon_{j}\right)}\right|^{5 / 3}+|\nabla \Pi|^{5 / 4} d x d t>\epsilon_{j}^{135 / 104} \varepsilon_{1},
$$

where $\tilde{B}_{i}(\mu):=B\left(x_{i}, \mu\right)$.

Combining the estimates above, we conclude that

$$
\sum_{i=1}^{N\left(\mathcal{S}, \epsilon_{j}\right)} \int_{t_{i}-\epsilon_{j}^{2}}^{t_{i}} \int_{\tilde{B}_{i}\left(\epsilon_{j}\right)}|\nabla u|^{2}+|u|^{10 / 3}+\left|\Pi-\bar{\Pi}_{\tilde{B}_{i}\left(\epsilon_{j}\right)}\right|^{5 / 3}+|\nabla \Pi|^{5 / 4} d x d t>\epsilon_{j}^{135 / 104-d} \varepsilon_{1} .
$$

With the help of (1.2), we know that the left hand side of the above equality is bounded by the initial data with finite-kinetic energy. Since $d>135 / 104$, we get a contradiction as $j \rightarrow \infty$ and thus complete the proof of Theorem 1.1. 
The remainder of this paper unfolds as follows. In Section 2, we recall the definition of suitable weak solutions to the Navier-Stokes equations. Then we will list some crucial bounds for the scaling invariant quantities. Section 3 contains the proof of Theorem 1.2,

Notations: Throughout this paper, we denote

$$
\begin{array}{lll}
B(x, \mu):=\left\{y \in \mathbb{R}^{3}|| x-y \mid \leq \mu\right\}, & B(\mu):=B(0, \mu), & \tilde{B}(\mu):=B\left(x_{0}, \mu\right), \\
Q(x, t, \mu):=B(x, \mu) \times\left(t-\mu^{2}, t\right), & Q(\mu):=Q(0,0, \mu), & \tilde{Q}(\mu):=Q\left(x_{0}, t_{0}, \mu\right) .
\end{array}
$$

The classical Sobolev norm $\|\cdot\|_{H^{s}}$ is defined as $\|f\|_{H^{s}}^{2}=\int_{\mathbb{R}^{n}}(1+|\xi|)^{2 s}|\hat{f}(\xi)|^{2} d \xi, s \in \mathbb{R}$. We denote by $\dot{H}^{s}$ homogenous Sobolev spaces with the norm $\|f\|_{\dot{H}^{s}}^{2}=\int_{\mathbb{R}^{n}}|\xi|^{2 s}|\hat{f}(\xi)|^{2} d \xi$. For $q \in[1, \infty]$, the notation $L^{q}(0, T ; X)$ stands for the set of measurable functions on the interval $(0, T)$ with values in $X$ and $\|f(t, \cdot)\|_{X}$ belongs to $L^{q}(0, T)$. For simplicity, we write

$$
\|f\|_{L^{q, \ell}(Q(\mu))}:=\|f\|_{L^{q}\left(-\mu^{2}, 0 ; L^{\ell}(B(\mu))\right)} \quad \text { and } \quad\|f\|_{L^{q}(Q(\mu))}:=\|f\|_{L^{q, q}(Q(\mu))} .
$$

Denote the average of $f$ on the set $\Omega$ by $\bar{f}_{\Omega}$. For convenience, $\bar{f}_{r}$ represents $\bar{f}_{B(r)}$. We will use the summation convention on repeated indices. $C$ is an absolute constant which may be different from line to line unless otherwise stated in this paper.

\section{Preliminaries}

We start with the definition of the suitable weak solution to the Navier-Stokes equations (1.1).

Definition 2.1. A pair $(u, \Pi)$ is called a suitable weak solution to the Navier-Stokes equations (1.1) provided the following conditions are satisfied,

(1) $u \in L^{\infty}\left(-T, 0 ; L^{2}\left(\mathbb{R}^{3}\right)\right) \cap L^{2}\left(-T, 0 ; \dot{H}^{1}\left(\mathbb{R}^{3}\right)\right), \Pi \in L^{3 / 2}\left(-T, 0 ; L^{3 / 2}\left(\mathbb{R}^{3}\right)\right)$.

(2) $(u, \Pi)$ solves (1.1) in $\mathbb{R}^{3} \times(-T, 0)$ in the sense of distributions;

(3) $(u, \Pi)$ satisfies the following inequality, for a.e. $t \in[-T, 0]$,

$$
\begin{aligned}
& \int_{\mathbb{R}^{3}}|u(x, t)|^{2} \phi(x, t) d x+2 \int_{-T}^{t} \int_{\mathbb{R}^{3}}|\nabla u|^{2} \phi d x d s \\
\leq & \int_{-T}^{t} \int_{\mathbb{R}^{3}}|u|^{2}\left(\partial_{s} \phi+\Delta \phi\right) d x d s+\int_{-T}^{t} \int_{\mathbb{R}^{3}} u \cdot \nabla \phi\left(|u|^{2}+2 \Pi\right) d x d s,
\end{aligned}
$$

where non-negative function $\phi(x, s) \in C_{0}^{\infty}\left(\mathbb{R}^{3} \times(-T, 0)\right)$.

In the light of the natural scaling property of Navier-Stokes equations, we introduce the following dimensionless quantities:

$$
\begin{array}{ll}
E(u, \mu)=\mu^{-1}\|u\|_{L^{\infty, 2}(Q(\mu))}^{2}, & E_{*}(\nabla u, \mu)=\mu^{-1}\|\nabla u\|_{L^{2}(Q(\mu))}^{2}, \\
E_{p}(u, \mu)=\mu^{p-5}\|u\|_{L^{p}(Q(\mu))}^{p}, & P_{5 / 4}(\nabla \Pi, \mu)=\mu^{-5 / 4}\|\nabla \Pi\|_{L^{5 / 4}(Q(\mu))}^{5 / 4}, \\
P_{3 / 2}(\Pi, \mu)=\mu^{-2}\left\|\Pi-\bar{\Pi}_{B(\mu)}\right\|_{L^{3 / 2}(Q(\mu))}^{3 / 2}, & P_{5 / 3}(\Pi, \mu)=\mu^{-5 / 3}\left\|\Pi-\bar{\Pi}_{B(\mu)}\right\|_{L^{5 / 3}(Q(\mu))}^{5 / 3}
\end{array}
$$


We recall the following lemma involving interpolation inequality. It is worth pointing out that the first part slightly improves the corresponding result obtained in [1, 12, 14]. As stated in Remark 1.4, we will utilize the second one, which was usually used for improving the Caffarelli-Kohn-Nirenberg theorem by a logarithmic factor in [2, 3, 16]. To make our paper more self-contained and more readable, we outline the proof of its general case (2.4).

Lemma 2.1. For $0<\mu \leq \frac{1}{2} \rho$ and $4 \leq b \leq 6$, there is an absolute constant $C$ independent of $\mu$ and $\rho$, such that

$$
\begin{aligned}
& E_{3}(u, \mu) \leq C\left(\frac{\rho}{\mu}\right)^{3 / 2} E^{3 / 4}(u, \rho) E_{*}^{3 / 4}(\nabla u, \rho)+C\left(\frac{\mu}{\rho}\right)^{3} E^{3 / 2}(u, \rho), \\
& E_{3}(u, \mu) \leq C\left(\frac{\rho}{\mu}\right)^{3 / 2} E^{1 / 2}(u, \rho) E_{*}(\nabla u, \rho)+C\left(\frac{\mu}{\rho}\right)^{3} E^{3 / 2}(u, \rho), \\
& E_{3}(u, \mu) \leq C\left(\frac{\rho}{\mu}\right)^{3 / 2} E^{\frac{b-3}{b-2}}(u, \rho) E_{*}^{\frac{b}{2 b-4}}(\nabla u, \rho)+C\left(\frac{\mu}{\rho}\right)^{3} E^{3 / 2}(u, \rho) .
\end{aligned}
$$

Proof of inequality (2.4). Taking advantage of the triangle inequality, Hölder's inequality and the Poincaré-Sobolev inequality, for any $3<b \leq 6$, we know that

$$
\begin{aligned}
\int_{B(\mu)}|u|^{3} d x & \leq C \int_{B(\mu)}\left|u-\bar{u}_{\rho}\right|^{3} d x+C \int_{B(\mu)}\left|\bar{u}_{\rho}\right|^{3} d x \\
& \leq C\left(\int_{B(\mu)}\left|u-\bar{u}_{\rho}\right|^{2} d x\right)^{\frac{b-3}{b-2}}\left(\int_{B(\mu)}\left|u-\bar{u}_{\rho}\right|^{b} d x\right)^{\frac{1}{b-2}}+\frac{\mu^{3} C}{\rho^{\frac{9}{2}}}\left(\int_{B(\rho)}|u|^{2} d x\right)^{3 / 2} \\
& \leq C \mu^{\frac{6-b}{2 b-4}}\left(\int_{B(\rho)}|u|^{2} d x\right)^{\frac{b-3}{b-2}}\left(\int_{B(\mu)}\left|u-\bar{u}_{\rho}\right|^{6} d x\right)^{\frac{b}{6 b-12}}+\frac{\mu^{3} C}{\rho^{\frac{9}{2}}}\left(\int_{B(\rho)}|u|^{2} d x\right)^{3 / 2} \\
& \leq C \mu^{\frac{6-b}{2 b-4}}\left(\int_{B(\rho)}|u|^{2} d x\right)^{\frac{b-3}{b-2}}\left(\int_{B(\rho)}|\nabla u|^{2} d x\right)^{\frac{b}{2 b-4}}+\frac{\mu^{3} C}{\rho^{\frac{9}{2}}}\left(\int_{B(\rho)}|u|^{2} d x\right)^{3 / 2} .
\end{aligned}
$$

Integrating in time on $\left(-\mu^{2}, 0\right)$ this inequality and using the Hölder inequality, for any $b \geq 4$, we obtain

$$
\begin{aligned}
\iint_{Q(\mu)}|u|^{3} d x d t \leq & C \mu^{\frac{1}{2}}\left(\sup _{-\rho^{2} \leq t \leq 0} \int_{B(\rho)}|u|^{2} d x\right)^{\frac{b-3}{b-2}}\left(\iint_{Q(\rho)}|\nabla u|^{2} d x d t\right)^{\frac{b}{2 b-4}} \\
& +C \frac{\mu^{5}}{\rho^{\frac{9}{2}}}\left(\sup _{-\rho^{2} \leq t \leq 0} \int_{B(\rho)}|u|^{2} d x\right)^{3 / 2}
\end{aligned}
$$

which leads to the desired inequality

$$
E_{3}(u, \mu) \leq C\left(\frac{\rho}{\mu}\right)^{3 / 2} E^{\frac{b-3}{b-2}}(u, \rho) E_{*}^{\frac{b}{2 b-4}}(\nabla u, \rho)+C\left(\frac{\mu}{\rho}\right)^{3} E^{3 / 2}(u, \rho) .
$$

One can also establish the following decay estimate of pressure via the interior estimate of harmonic function; see also [1, 2, 12, 14, 27, 30, 31] for different versions. For its proof, we refer the reader to [24, Lemma 2.1, p.222]. Thanks to the pressure $\Pi$ in terms of $\nabla \Pi$ in equations, we can invoke this lemma in the proof of Theorem 1.2 . 
Lemma 2.2. For $0<\mu \leq \frac{1}{2} \rho$, there is an absolute constant $C$ independent of $\mu$ and $\rho$ such that

$$
P_{3 / 2}(\Pi, \mu) \leq C\left(\frac{\rho}{\mu}\right)^{2} E_{3}(u, \rho)+C\left(\frac{\mu}{\rho}\right)^{5 / 2} P_{3 / 2}(\Pi, \rho) .
$$

Note that if the pair $(u, \Pi)$ is a suitable weak solution, so is $(u, \Pi-\bar{\Pi})$, therefore, the following $\varepsilon$-regularity criterion proved in [12, 14] is valid.

Proposition 2.3. Let $(u, \Pi)$ be a suitable weak solution to (1.1) in $Q(1)$. There exists $\varepsilon_{0}>0$ such that if

$$
\frac{1}{r^{2}} \iint_{Q(r)}|u|^{3}+\left|\Pi-\bar{\Pi}_{r}\right|^{3 / 2} d x d t \leq \varepsilon_{0},
$$

then $u$ is regular in $Q(r / 2)$.

\section{Proof of Theorem 1.2}

In this section, following the pathway of [9] together with the auxiliary lemmas in Section 2, we present the proof of Theorem 1.2.

Proof of Theorem 1.2. Without loss of generality, we assume that $\left(x_{0}, t_{0}\right)=(0,0)$. As [9], we present the assumption below

$$
\iint_{Q(2 \rho)}|\nabla u|^{2}+|u|^{10 / 3}+\left|\Pi-\bar{\Pi}_{2 \rho}\right|^{5 / 3}+|\nabla \Pi|^{5 / 4} d x d t \leq(2 \rho)^{5 / 3-\gamma} \varepsilon_{1},
$$

hence, it suffices to prove that $\gamma<115 / 312$ and $\gamma$ will be sufficiently close to $115 / 312$.

First, we assert that $E(u, \rho) \leq C \varepsilon_{1}^{3 / 5} \rho^{-\frac{3 \gamma}{5}}$. Indeed, letting $\phi(x, t)$ be a smooth positive function supported in $Q(2 \rho)$ and with value 1 on the ball $Q(\rho)$, then, employing the divergence free condition, Hölder's inequality thrice and the Gagliardo-Nirenberg inequality, we derive that

$$
\begin{aligned}
\iint_{Q(2 \rho)}\left(|u|^{2}-\overline{|u|^{2}}{ }_{2 \rho}\right) u \nabla \phi d x d t & \leq C \rho^{-1}\left\||u|^{2}-\overline{|u|^{2}}{ }_{2 \rho}\right\|_{L^{10 / 7,15 / 8}(Q(2 \rho))}\|u\|_{L^{10 / 3,15 / 7}(Q(2 \rho))} \\
& \leq C \rho^{-1}\left\|u^{2}\right\|_{L^{5 / 3}(Q(2 \rho))}^{1 / 2}\|u \nabla u\|_{L^{5 / 4}(Q(2 \rho))}^{1 / 2}\|u\|_{L^{10 / 3,15 / 7}(Q(2 \rho))} \\
& \leq C \rho^{-1 / 2}\|u\|_{L^{10 / 3}(Q(2 \rho))}^{5 / 2}\|\nabla u\|_{L^{2}(Q(2 \rho))}^{1 / 2}
\end{aligned}
$$

and

$$
\begin{aligned}
\iint_{Q(2 \rho)}( & \left.\Pi-\bar{\Pi}_{2 \rho}\right) u \nabla \phi d x d t \\
& \leq C \rho^{-1}\left\|\Pi-\bar{\Pi}_{2 \rho}\right\|_{L^{10 / 7,15 / 8}(Q(2 \rho))}\|u\|_{L^{10 / 3,15 / 7}(Q(2 \rho))} \\
& \leq C \rho^{-1}\left\|\Pi-\bar{\Pi}_{2 \rho}\right\|_{L^{5 / 3}(Q(2 \rho))}^{1 / 2}\|\Pi-\bar{\Pi}\|_{L^{5 / 4,15 / 7}(Q(2 \rho))}^{1 / 2}\|u\|_{L^{10 / 3,15 / 7}(Q(2 \rho))} \\
& \leq C \rho^{-1 / 2}\left\|\Pi-\bar{\Pi}_{2 \rho}\right\|_{L^{5 / 3}}^{1 / 2}\|\nabla \Pi\|_{L^{5 / 4}(Q(2 \rho))}^{1 / 2}\|u\|_{L^{10 / 3}(Q(2 \rho))} .
\end{aligned}
$$


These inequalities combined with the local energy inequality (2.1) leads to

$$
\begin{aligned}
& \sup _{-\rho^{2} \leq t<0} \int_{B(\rho)}|u|^{2} d x+2 C \iint_{Q(\rho)}|\nabla u|^{2} d x d t \\
& \leq C\left(\iint_{Q(2 \rho)}|u|^{10 / 3} d x d t\right)^{3 / 5}+C \rho^{-1 / 2}\|u\|_{L^{10 / 3}(Q(2 \rho))}^{5 / 2}\|\nabla u\|_{L^{2}(Q(2 \rho))}^{1 / 2} \\
&+C \rho^{-1 / 2}\|u\|_{L^{10 / 3}(Q(2 \rho))}\left\|\Pi-\bar{\Pi}_{2 \rho}\right\|_{L^{5 / 3}(Q(2 \rho))}^{1 / 2}\|\nabla \Pi\|_{L^{5 / 4}(Q(2 \rho))}^{1 / 2} \\
& \leq C \varepsilon_{1}^{3 / 5} \rho^{1-\frac{3 \gamma}{5}}
\end{aligned}
$$

where we have used (3.1) and assumed that $\gamma \leq 5 / 12$. As a consequence,

$$
E(u, \rho) \leq C \varepsilon_{1}^{3 / 5} \rho^{-\frac{3 \gamma}{5}} .
$$

Second, iterating (2.5) in Lemma 2.2, we infer that

$$
P_{3 / 2}\left(\Pi, \theta^{N} \mu\right) \leq C \sum_{k=1}^{N} \theta^{-2+\frac{5(k-1)}{2}} E_{3}\left(u, \theta^{N-k} \mu\right)+C \theta^{5 N / 2} P_{3 / 2}(\Pi, \mu) .
$$

In view of the Poincaré-Sobolev inequality and Hölder's inequality, we see that

$$
\begin{aligned}
\left\|\Pi-\bar{\Pi}_{\mu}\right\|_{L^{3 / 2}(Q(\mu))}^{3 / 2} & \leq\left\|\Pi-\bar{\Pi}_{\mu}\right\|_{L^{5 / 4,15 / 7}(Q(\mu))}^{1 / 2}\left\|\Pi-\bar{\Pi}_{\mu}\right\|_{L^{5 / 3,30 / 23}(Q(\mu))} \\
& \leq C \rho^{1 / 2}\|\nabla \Pi\|_{L^{5 / 4}(Q(\mu))}^{1 / 2}\left\|\Pi-\bar{\Pi}_{\mu}\right\|_{L^{5 / 3}(Q(\mu))} .
\end{aligned}
$$

Dividing both sides of (3.5) by $\mu^{2}$, we have

$$
P_{3 / 2}(\Pi, \mu) \leq C P_{5 / 4}^{2 / 5}(\nabla \Pi, \mu) P_{5 / 3}^{3 / 5}(\Pi, \mu) .
$$

By inserting the above inequality into (3.4), we know that

$$
P_{3 / 2}\left(\Pi, \theta^{N} \mu\right) \leq C \sum_{k=1}^{N} \theta^{-2+\frac{5(k-1)}{2}} E_{3}\left(u, \theta^{N-k} \mu\right)+C \theta^{5 N / 2} P_{5 / 4}^{2 / 5}(\nabla \Pi, \mu) P_{5 / 3}^{3 / 5}(\Pi, \mu) .
$$

To proceed further, we set $r=\rho^{\alpha}=\theta^{N} \mu, \theta=\rho^{\beta}, r_{N}=\mu=\theta^{-N} r=\rho^{\alpha-N \beta}$, where $\alpha, \beta$ is determined by $\gamma$. Their precise selection will be given in the end. Hence, we derive from (3.6) that

$$
\begin{aligned}
P_{3 / 2}(\Pi, r)+E_{3}(u, r) & \leq C \sum_{k=1}^{N} \theta^{-2+\frac{5(k-1)}{2}} E_{3}\left(u, \theta^{-k} r\right)+C \theta^{5 N / 2} P_{5 / 4}^{2 / 5}\left(\nabla \Pi, r_{N}\right) P_{5 / 3}^{3 / 5}\left(\Pi, r_{N}\right) \\
& =C \sum_{k=1}^{N} \theta^{-2+\frac{5(k-1)}{2}} E_{3}\left(u, r_{k}\right)+C \theta^{5 N / 2} P_{5 / 4}^{2 / 5}\left(\nabla \Pi, r_{N}\right) P_{5 / 3}^{3 / 5}\left(\Pi, r_{N}\right),
\end{aligned}
$$

where we have used the fact that $E_{3}(u, r) \leq C \theta^{-2} E_{3}\left(u, \theta^{-1} r\right)$. Our aim below is to resort Proposition 2.3 to complete the proof. To this end, we suppose that $r_{N} \leq \rho$, then, we can adopt (2.3) in Lemma 2.1, (3.3) and the hypothesis (3.1) to obtain

$$
\begin{aligned}
E_{3}\left(u, r_{k}\right) & \leq C\left(\frac{\rho}{r_{k}}\right)^{\frac{3}{2}} E^{1 / 2}(u, \rho) E_{*}(\nabla u, \rho)+\left(\frac{r_{k}}{\rho}\right)^{3} E^{3 / 2}(u, \rho) \\
& \leq C \varepsilon_{1}^{9 / 10}\left(\rho^{\frac{13}{6}-\frac{3}{2}(\alpha-k \beta)-\frac{13 \gamma}{10}}+\rho^{3 \alpha-3-3 k \beta-\frac{9 \gamma}{10}}\right) .
\end{aligned}
$$


Therefore, for sufficiently large $N$, some elementary calculations yield

$$
\begin{aligned}
\sum_{k=1}^{N} \theta^{-2+\frac{5(k-1)}{2}} E_{3}\left(u, r_{k}\right) & \leq C \varepsilon_{1}^{9 / 10} \sum_{k=1}^{N}\left(\rho^{4 k \beta-\frac{9 \beta}{2}+\frac{13}{6}-\frac{3 \alpha}{2}-\frac{13 \gamma}{10}}+\rho^{-\frac{k \beta}{2}-\frac{9 \beta}{2}+3 \alpha-3-\frac{9 \gamma}{10}}\right) \\
& \leq C \varepsilon_{1}^{9 / 10}\left(\rho^{-\frac{\beta}{2}+\frac{13}{6}-\frac{3 \alpha}{2}-\frac{13 \gamma}{10}}+\rho^{-\frac{N \beta}{2}-\frac{9 \beta}{2}+3 \alpha-3-\frac{9 \gamma}{10}}\right) \\
& \leq C \varepsilon_{1}^{9 / 10} \rho^{-\frac{11 \beta}{6}+\frac{4}{9}-\frac{7 \gamma}{6}-\frac{N \beta}{6}}
\end{aligned}
$$

where $\alpha$ is determined from

$$
-\frac{\beta}{2}+\frac{13}{6}-\frac{3 \alpha}{2}-\frac{13 \gamma}{10}=-\frac{N \beta}{2}-\frac{9 \beta}{2}+3 \alpha-3-\frac{9 \gamma}{10} .
$$

In short,

$$
\alpha=\frac{2}{9}\left(4 \beta+\frac{31}{6}-\frac{2 \gamma}{5}+\frac{1}{2} N \beta\right)
$$

According to $r_{N} \leq \rho$ assumed above, namely,

$$
\rho^{\alpha-N \beta} \leq \rho,
$$

(3.1) and (3.7), we get

$$
\begin{aligned}
\theta^{5 N / 2} P_{5 / 4}^{2 / 5}( & \left.\nabla \Pi, r_{N}\right) P_{5 / 3}^{3 / 5}\left(\Pi, r_{N}\right) \\
& \leq \rho^{\frac{5 N \beta}{2}} r_{N}^{-\frac{3}{2}}\left(\iint_{Q(2 \rho)}|\nabla \Pi|^{5 / 4} d x d t\right)^{2 / 5}\left(\iint_{Q\left(r_{N}\right)}\left|\Pi-\bar{\Pi}_{2 \rho}\right|^{5 / 3} d x d t\right)^{3 / 5} \\
& \leq \rho^{\frac{5 N \beta}{2}} r_{N}^{-\frac{3}{2}}\left(\iint_{Q(2 \rho)}|\nabla \Pi|^{5 / 4} d x d t\right)^{2 / 5}\left(\iint_{Q(2 \rho)}\left|\Pi-\bar{\Pi}_{2 \rho}\right|^{5 / 3} d x d t\right)^{3 / 5} \\
& \leq C \rho^{\frac{23}{6} N \beta-\frac{4 \beta}{3}-\frac{9 \gamma}{10}} \varepsilon_{1} .
\end{aligned}
$$

To conclude, by Proposition 2.3, we need $-\frac{11 \beta}{6}+\frac{4}{9}-\frac{7 \gamma}{6}-\frac{N \beta}{6} \geq 0$ and $\frac{23}{6} N \beta-\frac{4 \beta}{3}-\frac{9 \gamma}{10} \geq 0$, moreover, from (3.8), we know that $\alpha-N \beta-1 \geq 0$.

In summary, the index $\gamma$ should satisfy

$$
\gamma \leq \min \left\{\frac{115 N \beta-40 \beta}{27}, \frac{8-3 N \beta-33 \beta}{21}, \frac{5-30 N \beta+30 \beta}{3}, \frac{5}{12}\right\},
$$

which means that $N \beta=9 / 104$ is appropriate.

Eventually, for any fixed $\gamma<115 / 312$, we choose $N$ sufficiently large such that

$$
\beta=\frac{9}{104 N} \leq \frac{7}{11}\left(\frac{115}{312}-\gamma\right)
$$

Then, we select a fixed $\rho<1$ such that $\rho^{\beta}<1 / 2$. With $\gamma, N, \beta$ in hand, we pick $\alpha=$ $\frac{2}{9}\left(4 \beta-\frac{2 \gamma}{5}+\frac{3251}{624}\right)$. This allows us to get

$$
P_{3 / 2}(\Pi, r)+E_{3}(u, r) \leq C \varepsilon_{1}^{9 / 10} \leq \varepsilon_{0},
$$

with $r=\rho^{\alpha}$. This completes the proof of Theorem 1.2, 


\section{Acknowledgement}

The authors would like to express their deepest gratitude to two anonymous referees and the editors for careful reading of our manuscript, the invaluable comments and suggestions which helped to improve the paper greatly, especially, enlightening comments on inequality (3.6). In addition, we would also like to express our thanks to Prof. Minsuk Yang for pointing out that the proof of (2.2) in Lemma 2.1 can be found in [3]. Wang was partially supported by NSFC (No. 11601492). Wu was partially supported by NSFC (No.11101405).

\section{References}

[1] L. Caffarelli, R. Kohn and L. Nirenberg, Partial regularity of suitable weak solutions of Navier-Stokes equation, Comm. Pure. Appl. Math., 35 (1982), 771-831.

[2] H. Choe, and J. Lewis, On the singular set in the Navier-Stokes equations, J. Funct. Anal. 175 (2000) 348-369.

[3] H. Choe and M. Yang, Hausdorff measure of the singular set in the incompressible magnetohydrodynamic equations, Comm. Math. Phys. 336 (2015) 171-198.

[4] K. Choi and A. Vasseur, Estimates on fractional higher derivatives of weak solutions for the Navier-Stokes equations. Ann. Inst. H. Poincaré Anal. Non Linéaire, 31 (2014), 899-945.

[5] P. Constantin, Navier-Stokes equations and area of interfaces, Comm. Math. Phys., 129 (1990), 241-266.

[6] K. Falconer, Fractal Geometry: Mathematical Foundations and Applications (New York: Wiley) 1990.

[7] C. Foias, C. Guillopé and R. Temam, New a priori estimates for Navier-Stokes equations in Dimension 3, Comm. Partial Diff. Equat., 6 (1981), 329-359.

[8] Y. Giga and H. Sohr, Abstract $L^{p}$-estimates for the Cauchy problem with applications to the Navier-Stokes equations in exterior domains, J. Funct. Anal., 102 (1991), 72-94.

[9] Y. Koh and M. Yang, The Minkowski dimension of interior singular points in the incompressible Navier-Stokes equations. J. Differential Equations., 261 (2016), 31373148 .

[10] I. Kukavica, The fractal dimension of the singular set for solutions of the Navier-Stokes system Nonlinearity., 22 (2009), 2889-2900.

[11] I. Kukavica and Y. Pei, An estimate on the parabolic fractal dimension of the singular set for solutions of the Navier-Stokes system. Nonlinearity., 25 (2012), 2775-2783.

[12] O. Ladyzenskaja and G. Seregin, On partial regularity of suitable weak solutions to the three-dimensional Navier-Stokes equations, J. Math. Fluid Mech., 1 (1999), 356-387.

[13] J. Leray, Sur le mouvement déun liquide visqueux emplissant léspace, Acta Math., 63 (1934), 193-248. 
[14] F. Lin, A new proof of the Caffarelli-Kohn-Nirenberg Theorem, Comm. Pure Appl. Math., 51 (1998), 241-257.

[15] W. Ren, Y. Wang and G. Wu, Partial regularity of suitable weak solutions to the multi-dimensional generalized magnetohydrodynamics equations. Commun. Contemp. Math., (2016). Doi: 10.1142/S0219199716500188.

[16] - General logarithmic improvement on the Caffarelli-Kohn-Nirenberg theorem, submitted for publication. 2016.

[17] J. Robinson and W. Sadowski, Decay of weak solutions and the singular set of the three-dimensional Navier-Stokes equations, Nonlinearity., 20 (2007), 1185-1191.

[18] _ _ Almost-everywhere uniqueness of Lagrangian trajectories for suitable weak solutions of the three-dimensional Navier-Stokes equations, Nonlinearity., 22, (2009) 2093-2099.

[19] - On the Dimension of the Singular Set of Solutions to the Navier-Stokes Equations, Comm. Math. Phys., 309 (2012), 497-506.

[20] V. Scheffer, Turbulence and Hausdorff dimension, in Turbulence and the Navier-Stokes Equations, Lecture Notes in Math., Springer-Verlag, 565 (1976), 94-112.

[21] _ Partial regularity of solutions to the Navier-Stokes equations, Pacific J. Math., 66 (1976), 535-552.

[22] _ Hausdorff measure and the Navier-Stokes equations, Comm. Math. Phys., 55 (1977), 97-112.

[23] The Navier-Stokes equations in space dimension four, Comm. Math. Phys., 61 (1978), 41-68.

[24] G. Seregin, On smoothness of $L_{3, \infty}$-solutions to the Navier-Stokes equations up to boundary. Math. Ann., 332 (2005), 219-238.

[25] V. A. Solonnikov. Estimates for solutions of nonstationary Navier-Stokes equations. J. Soviet Math., 8 (1977), 467-523.

[26] L. Tang and Y. Yu, Partial regularity of suitable weak solutions to the fractional Navier-Stokes equations. Comm. Math. Phys., 334 (2015), 1455-1482.

[27] G. Tian and Z. Xin, Gradient estimation on Navier-Stokes equations, Comm. Anal. Geom., 7 (1999), 221-257.

[28] A. Vasseur, A new proof of partial regularity of solutions to Navier-Stokes equations, NoDEA Nonlinear Differential Equations Appl., 14 (2007), 753-785.

[29] Higher derivatives estimate for the 3D Navier-Stokes equation. Ann. Inst. H. Poincaré Anal. Non Linéaire, 27 (2010), 1189-1204.

[30] W. Wang and Z. Zhang, On the interior regularity criteria and the number of singular points to the Navier-Stokes equations. J. Anal. Math., 123 (2014), 139-170.

[31] Y. Wang and G. Wu, A unified proof on the partial regularity for suitable weak solutions of non-stationary and stationary Navier-Stokes equations. J. Differential Equations., 256 (2014), 1224-1249. 